\title{
Digestibilidad in vivo de morera (Morus alba), con diferentes niveles de concentrado en curies (Cavia porcellus)
}

\author{
In vivo digestibility of morera (Morus alba), with different levels of \\ concentrate in guinea pig (Cavia porcellus)
}

\section{Digestibilidade in vivo da amoreira (Morus alba), com diferentes níveis de concentrado em cobaia (Cavia porcellus)}

\author{
Daniel Enrique Villegas González ${ }^{1}$ y María Ligia Roa Vega ${ }^{2}$ \\ ${ }^{1}$ Medico Veterinario Zootecnista, Universidad de los Llanos y \\ 2Zootecnista, Esp, MSc, Directora Grupo de Investigación Agroforestería, Docente \\ Universidad de los Llanos \\ daniel.villegas@unillanos.edu.co
}

Recibido 20 de septiembre 2020, aceptado 16 noviembre 2020

\section{RESUMEN}

La carne de cuy (Cavia porcellus) tiene una elevada demanda en Ecuador, Perú y Colombia principalmente en Pasto. Se ha evidenciado que su consumo se ha extendido hacia gran parte del país, existen pocos estudios que involucren estos animales y mucho menos evalúen las estrategias alimenticias en su comportamiento en cuanto a metabolismo y aprovechamiento de nutrientes. De la misma manera no se conocen alternativas teniendo en cuenta que esta especie consume gran cantidad de forrajes, lo que favorece por la disminución en costos de producción de carne, evitando el uso de concentrado al $100 \%$, puesto que este insumo tiene un costo elevado, además, influye de manera negativa en sabor de la carne. El presente estudio se realizó en el municipio de Villavicencio, con una altitud de 465 metros sobre el nivel del mar, temperatura promedio de $27^{\circ} \mathrm{C}$ y precipitación anual entre 1900 y 3250 milímetros. Los cuyes se fueron distribuidos en cuatro tratamientos con tres repeticiones y cada una con dos unidades experimentales, para un total de 24 animales con un peso promedio $1027.5 \pm 19 \mathrm{~g}$ distribuidos en un diseño completamente al azar, los cuales se alojaron en jaulas y sometidos a un periodo de acostumbramiento de cinco días, y cuatro de toma de datos que se 
realizó a partir del día quinto. Para cada repetición se tomaron cuatro muestras, las cuales fueron evaluadas en el Laboratorio de Nutrición Animal de la Universidad de los Llanos. Las dietas suministradas por día a todos los tratamientos fueron morera fresca (MF) ad libitum, suplementados de la siguiente manera: T1 con concentrado comercial (CC), $1 \%$ de su peso vivo (PV); T2: CC, $2 \%$ de su PV; T3: CC $3 \%$ de su PV y T4: únicamente MF. Se realizaron análisis nutricionales a las dietas y excretas. Las variables a evaluar fueron coeficientes de digestibilidad (Cod) de la materia seca (MS), proteína, grasa, extracto no nitrogenado (ENN), fibra cruda (FC), \% nutrientes digestibles totales (NDT); cálculos de: Energía digestible (ED) y energía metabolizarle (EM), Cod: método que valora el consumo y la excreción de los nutrientes para determinar el aprovechamiento en el tracto digestivo. El estudio fue importante en el sentido de que se estimaron valores de referencia para el sector en términos de digestibilidad de la morera y el concentrado comercial en cuyes. Se determinó que las dietas de mejor comportamiento $(\mathrm{P}<0.05)$, fueron $\mathrm{CC}$ al $2 \%$ (T2) y $3 \%$ (T3) del PV, a pesar que la que mayores valores de digestibilidad obtenidos fue para T3, el T2 observó un comportamiento similar según el estudio, pudiéndose utilizar sin disminuir drásticamente la digestibilidad y ahorrando un $1 \%$ de concentrado por animal representado un ahorro importante.

Palabras clave: Arbustivas, alimentación, Cavia porcellus.

\section{ABSTRACT}

Guinea pig (Cavia porcellus) meat is in high demand in Ecuador, Peru and Colombia, mainly in Pasto. It has been shown that their consumption has spread to a large part of the country, there are few studies that involve these animals and much less evaluate the nutritional strategies in their behavior in terms of metabolism and use of nutrients. In the same way, there are no known alternatives, taking into account that this species consumes a large amount of forage, which favors the decrease in meat production costs, avoiding the use of $100 \%$ concentrate, since this input has a high cost. In addition, it has a negative influence on the flavor of the meat. The present study was carried out in the municipality of Villavicencio, with an altitude of 465 meters above sea level, an average temperature of $27^{\circ} \mathrm{C}$ and annual rainfall 
between 1900 and 3250 millimeters. The guinea pigs were distributed in four treatments with three repetitions and each one with two experimental units, for a total of 24 animals with an average weight of $1027.5 \pm 19 \mathrm{~g}$ distributed in a completely random design, which were housed in cages and subjected to a period of acclimatization of five days, and four days of data collection that was carried out from the fifth day. For each repetition, four samples were taken, which were evaluated in the Animal Nutrition Laboratory of the University of Los Llanos. The diets supplied per day to all the treatments were fresh mulberry (FM) ad libitum, supplemented as follows: T1 with commercial concentrate (CC), $1 \%$ of its live weight (LW); T2: CC, $2 \%$ of your LW; T3: CC $3 \%$ of your LW and T4: FM only. Nutritional analyzes were carried out on the diets and excreta. The variables to evaluate were digestibility coefficients (Cod) of dry matter (DM), protein, fat, non-nitrogenous extract (NNE), crude fiber (CF), \% total digestible nutrients (TDN); calculations of: digestible energy $(\mathrm{DE})$ and metabolized energy (ME), Cod: method that assesses the consumption and excretion of nutrients to determine the use in the digestive tract. The study was important in the sense that reference values were estimated for the sector in terms of digestibility of mulberry and commercial concentrate in guinea pigs. It was determined that the diets with the best performance $(\mathrm{P}<0.05)$ were $\mathrm{CC}$ at $2 \%(\mathrm{~T} 2)$ and $3 \%$ (T3) of the LW, although the one with the highest digestibility values obtained was for T3, the T2 observed a similar behavior according to the study, being able to use without drastically reducing digestibility and saving $1 \%$ of concentrate per animal representing a significant saving.

Keywords: Shrubby, feeding, Cavia porcellus.

\section{RESUMO}

A carne de cobaias (Cavia porcellus) tem alta demanda no Equador, Peru e Colômbia, principalmente em Pasto. Está comprovado que seu consumo se espalhou por grande parte do país, poucos são os estudos que envolvem esses animais e muito menos avaliam as estratégias nutricionais em seu comportamento quanto ao metabolismo e uso de nutrientes. Da mesma forma, não existem alternativas conhecidas, visto que esta espécie consome grande quantidade de 
forragem, o que favorece a diminuição dos custos de produção de carne, evitando o uso de concentrado $100 \%$, visto que este insumo possui alto custo. Além disso, influencia negativamente 0 sabor da carne. O presente estudo foi realizado no município de Villavicencio, com altitude de 465 metros acima do nível do mar, temperatura média de $27^{\circ} \mathrm{C}$ e pluviosidade anual entre 1900 e 3250 milímetros. As cobaias foram distribuídas em quatro tratamentos com três repetições e cada um com duas unidades experimentais, totalizando 24 animais com peso médio de $1027.5 \pm 19 \mathrm{~g}$ distribuídos em delineamento inteiramente ao acaso, os quais foram alojados em gaiolas e submetido a um período de aclimatação de cinco dias, sendo quatro dias de coleta de dados que foi realizada a partir do quinto dia. Para cada repetição, foram retiradas quatro amostras, as quais foram avaliadas no Laboratório de Nutrição Animal da Universidade de Los Llanos. As dietas fornecidas por dia para todos os tratamentos foram amora in natura (AF) ad libitum, suplementada da seguinte forma: T1 com concentrado comercial (CC), $1 \%$ do seu peso vivo (PV); T2: CC, $2 \%$ do seu PV; T3: CC $3 \%$ de seu HP e T4: AF apenas. Análises nutricionais foram realizadas nas dietas e excrementos. As variáveis avaliadas foram: coeficientes de digestibilidade (Bacalhau) da matéria seca (MS), proteína, gordura, extrato não nitrogenado ( $E N N)$, fibra bruta $(F C)$, \% de nutrientes digestíveis totais (NDT); cálculos de: energia digestível (ED) e energia metabolizada (EM); Bacalhau: método que avalia o consumo e a excreção de nutrientes para determinar o uso no trato digestivo. $O$ estudo foi importante na medida em que foram estimados valores de referência para o setor em termos de digestibilidade da amora e do concentrado comercial em cobaias. Determinou-sé que as dietas com melhor desempenho $(\mathrm{P}<0.05)$ foram CC a $2 \%$ (T2) e $3 \%$ (T3) do PV, embora aquela com os maiores valores de digestibilidade obtidos tenha sido para T3, o T2 observado comportamento semelhante de acordo com o estudo, podendo utilizar sem reduzir drasticamente a digestibilidade e economizando $1 \%$ de concentrado por animal representando uma economia significativa.

Palavras-chave: Arbustivo, alimentação, Cavia porcellus. 


\section{INTRODUCCIÓN}

La carne de cuy (Cavia porcellus) tiene una elevada demanda en Ecuador, Perú y Colombia principalmente en Pasto. Se ha evidenciado que su consumo se ha extendido hacia gran parte del país, existen pocos estudios en el Meta que involucren estos animales y mucho menos evalúen las estrategias alimenticias y el comportamiento del animal en cuanto a metabolismo y aprovechamiento de nutrientes. De la misma manera no se conocen alternativas de alimentación teniendo en cuenta que esta especie consume gran cantidad de forrajes, lo que favorece que los costos de producción de carne sean bajos, evitando el uso de concentrado al $100 \%$, puesto que este insumo tiene un costo elevado. Además, estos alimentos influyen de manera negativa en sabor de la carne de cuy. Existen pocas experiencias de alimentación reportadas por la literatura que puedan ser utilizadas como opciones por parte del productor. En Colombia ha tomado gran acogida el consumo de carne de cuy presentándose así otra alternativa de seguridad alimentaria, la producción de carne de cuyes (Cavia porcellus) que, por su alto consumo de forrajes, da como resultado una de buena palatabilidad para los seres humanos. La cuyicultura ha crecido en los últimos años en Colombia y en el Meta, siendo este un animal que para su pequeña talla aporta gran cantidad de carne, sabor agradable, niveles elevados de proteína, sumado a esto se puede decir que tienen un crecimiento rápido, no requieren mucho espacio, siendo resistentes y tolerantes a los cambios de temperatura. Una de las producciones con más auge en la actualidad es la industria avícola la cual tiene una participación en el producto interno bruto agropecuario de $7.8 \%$ (Fedegan, 2011), sus costos de producción son muy elevados a tal punto de que el productor tenga que cumplir un mínimo de producción para justificar la inversión. Con respecto a sus valores nutricionales tiene $20 \%$ de proteína, $4 \%$ de grasa, calorías $388.9 \mathrm{kcal} / 100 \mathrm{~g}$ (Carvajal, 2001), en cuanto a la carne Santos, (2007) reporta que la carne de cuy es magra, es decir con un porcentaje de grasa menor al $10 \%$, con alto contenido de proteínas $(20.3 \%)$, baja en contenidos de colesterol $(65 \mathrm{mg} / 100 \mathrm{~g}$ ) y sodio, por lo que es ideal para incluirla en una alimentación variada y equilibrada, de la misma manera es una alternativa que ofrece parámetros saludables para diferentes poblaciones que no pueden 
consumir altos niveles de colesterol. La carne de cuy en Colombia tiene un valor elevado, por lo que en el momento de industrializar el producto no va a generar impacto negativo. El cuy se explota en la región Andina de Bolivia, Colombia, Ecuador y Perú; se adapta a diversas condiciones climáticas pudiendo encontrarse desde la costa o el llano hasta alturas de 4500 msnm y en zonas tanto frías como cálidas (Hurtado et al., 2012) de la misma manera los autores exponen que los cuyes carecen de las enzimas necesarias para convertir L-gulonolactona en ácido ascórbico, por eso, al ser alimentados con forrajes secos deben recibir una suplementación de vitamina $\mathrm{C}$. Es un animal pequeño muy dócil y fácil de manejar, herbívoro, mono gástrico, tiene un estómago donde inicia su digestión enzimática y un ciego funcional donde se realiza la fermentación bacteriana de forrajes y granos, tiene un aporte de proteína elevado, $20.3 \%$ y de grasa de 7.8\% (Sanchez, 2014). Con respecto a los parámetros reproductivos del animal Pajares, (2009) expone que las hembras son fértiles a la edad de 50 a 70 días y los machos a los 50 días; por eso hay que criarlos separados hasta la edad de reproducción, el período de gestación es de 68 días, a veces hasta con ocho crías por parto, su crecimiento del cuy es rápido durante las tres primeras semanas debido al valor nutricional de la leche materna y al consumo de alimento que inicia a las pocas horas del nacimiento, en virtud de estas características es posible realizar destetes precoces.

El término "digestibilidad del alimento o de la materia seca" es usado para describir la porción del alimento absorbido por el organismo que es medido como la diferencia entre el total de alimento ingerido y la cantidad de heces producidas en un tiempo determinado (Cruz et al., 2008).

El análisis del aprovechamiento de cada uno de los nutrientes en el organismo del animal se hace teniendo en cuenta su digestibilidad, esta valoración se consigue con diferentes métodos. La digestibilidad in vivo de un alimento se puede medir directa e indirectamente. En la forma directa se registra exactamente el consumo de alimento y la excreción fecal de un animal sometido a un tratamiento específico, en un período de tiempo dado. Como desventaja de este método, puede existir contaminación entre excretas con la orina; además el confinamiento de los animales 
reduce el tono muscular y probablemente disminuye el tránsito de ingesta, por lo tanto, se puede sobre-estimar la digestibilidad con respecto a los animales no alojados en jaulas. La forma indirecta para medir la digestibilidad no requiere cuantificar el consumo ni la excreción fecal, se puede utilizar marcadores inertes que no contengan ningún nutriente, se agregan incluyéndolos en el alimento (Perez et al., 2010). El desarrollo de la digestibilidad comparada permite encontrar la relación del aprovechamiento de la proteína en el animal, utilizando nuevas alternativas de alimentación y confrontándolas con el concentrado comercial comúnmente utilizado en la cunicultura.

Pond y Church, (1994) afirman que el método de los nutrientes digestibles totales (NDT) valora el alimento en su contenido de energía, partiendo de los cálculos de digestibilidad directa in vivo, donde se mide el nutriente consumido (NC) y el excretado (NE), realizando los análisis proximales para aplicar fórmulas y así determinar los coeficientes de digestibilidad de cada nutriente (cod). Al contenido de carbohidratos digestibles, llamado extracto no nitrogenado digestible (ENN), se le suma las fracciones de fibra, proteína y la grasa digestible (ésta última multiplicada por la constante 2.25, esto debido a su contenido. El valor energético relativo $100 \%$ de un ingrediente es equivalente $1 \mathrm{~kg}$ de ND, que a su vez es igual 4400 Kcal de energía digestible (ED) o a 3560 Kcal de energía metabolizable (EM).

La digestibilidad es uno de los factores más importantes para evaluar la calidad nutritiva de las raciones que consumen los animales domésticos, porque indica el grado en que los nutrientes de los ingredientes van a ser aprovechados directamente por el animal. Una buena digestibilidad de la dieta resultará en una mayor productividad por parte del animal. Existen diferentes maneras de determinar la digestibilidad de los nutrientes, tales como las pruebas de digestibilidad in vivo (método de colección total o parcial), digestibilidad in situ y digestibilidad in vitro (Barreyro, 2000). La morera se encuentra en alturas desde el nivel del mar hasta 4000 metros de altura, en zonas secas y húmedas, no es tolerante a suelos mal drenados y muy compactos (Benavides, 1995), el valor nutricional de la especie a trabajar juega un papel importante para el conocimiento del aprovechamiento por 
parte del animal y de la misma manera la evaluación de la calidad y aporte, en este orden de ideas (Medina et al., 2009), en una artículo de revisión recalca y reúne información referente a el aporte nutricional como recurso forrajero y sus usos, reporta que la morera se usa como fuente nutricional en alimentación humana por sus frutos con alta palatabilidad, de la misma manera se usa como alimento o sustrato para gusanos en producciones de hilos finos, tiene uso como fuente medicinal en diversos países. En cuanto a la composición química se reporta que la planta según su tiempo de corte aporta en promedio $16 \%$ de proteína verdadera, FDN 38\%, FDA 29\% y cenizas de 8\%, además se reporta altos contenidos de ácidos grasos esenciales (Garcia, 2005) en un estudio realizado con conejos evaluó el efecto de la incorporación de morera reemplazando un concentrado comercial se obtuvieron diferencias en la composición de ácido alfa-linolénico, el lomo de conejos alimentados con morera fue superior al grupo de conejos alimentados a voluntad, la misma tendencia se observó en pierna, con lo que se demuestra el potencial de la morera como fuente de ácido alfa-linolénico (n-3). El autor concluye que "el potencial de la especie para sistemas agropecuarios no es conocido", en igual medida, en todos los países de la región. Fuera de Centroamérica y el Caribe, el uso de la morera para la alimentación animal es más limitado; aspecto que se encuentra asociado con el poco conocimiento que existe sobre la especie y la incipiente divulgación que se le ha dado en Suramérica, excluyendo a Brasil. En este sentido, se debe de realizar un mayor número de estudios en dichas zonas, con la finalidad de trazar estrategas viables de uso, en función de las particularidades socioculturales de los productores y de aspectos inherentes a los sistemas de producción de cada país. Definitivamente existen muchos estudios en donde se estandariza y se conoce el comportamiento de esta planta, pero en el sector son muy pocos y asimismo son nulos los trabajos en morera para alimentación de cuyes siendo una de la principal justificación de investigación.

\section{METODOLOGÍA}

El presente estudio se realizó en el municipio de Villavicencio, en la granja de UNILLANOS sede Barcelona y en el Laboratorio de Nutrición Animal ubicados en el 
kilómetro doce vía Puerto López, con una altitud de 465 metros sobre el nivel del mar, temperatura promedio de $27^{\circ} \mathrm{C}$ y precipitación anual entre 1900 y 3250 milímetros.

Los cuyes se fueron distribuidos en cuatro tratamientos con tres repeticiones cada una con dos unidades experimentales, para un total de 24 animales con un peso promedio $1027.5 \pm 19 \mathrm{~g}$ en un diseño completamente al azar, los cuales se alojaron en jaulas y sometidos a un periodo de acostumbramiento de cinco días, y cuatro de toma de datos que se realizó a partir del día quinto. Para cada repetición se tomaron cuatro muestras, las cuales fueron evaluadas en el Laboratorio de Nutrición Animal de la Universidad de los Llanos (Figura 1). Las dietas suministradas por día a todos los tratamientos fueron morera fresca (MF) ad libitum, suplementados de la siguiente manera: T1: morera fresca (MF) ad libitum más $1 \%$ de su peso vivo (PV) de concentrado comercial (CC); T2: MF ad libitum más $2 \%$ de su peso vivo (PV) de CC; T3: MF ad libitum más $2 \%$ de su peso vivo (PV) de CC y T4: únicamente MF ad libitum. Los consumos y análisis nutricionales de los tratamientos experimentales se detallan en las Tablas 1 y 2 .

Se realizaron análisis nutricionales a las dietas y excretas. Las variables a evaluar fueron coeficientes de digestibilidad (Cod) de la materia seca (MS), proteína, grasa, extracto no nitrogenado (ENN), fibra cruda (FC), \% nutrientes digestibles totales (NDT); cálculos de: Energía digestible (ED) y energía metabolizarle (EM), Cod: Método que valora el consumo y la excreción de los nutrientes para determinar el aprovechamiento en el tracto digestivo, aplicando las siguientes fórmulas (Pond y Church, 1994):

Coeficiente de digestibilidad de un nutriente $(\operatorname{cod})=\frac{N C-N E}{N C} \times 100$

$N C=$ Nutriente consumido

$N E=$ Nutriente excretado

$\% N D T=\%$ proteina $x \operatorname{cod}+\% \operatorname{grasa} x \operatorname{cod} \times 2.25+\%$ fibra $x \operatorname{cod}+\%$ ENN $x \operatorname{cod}$ 


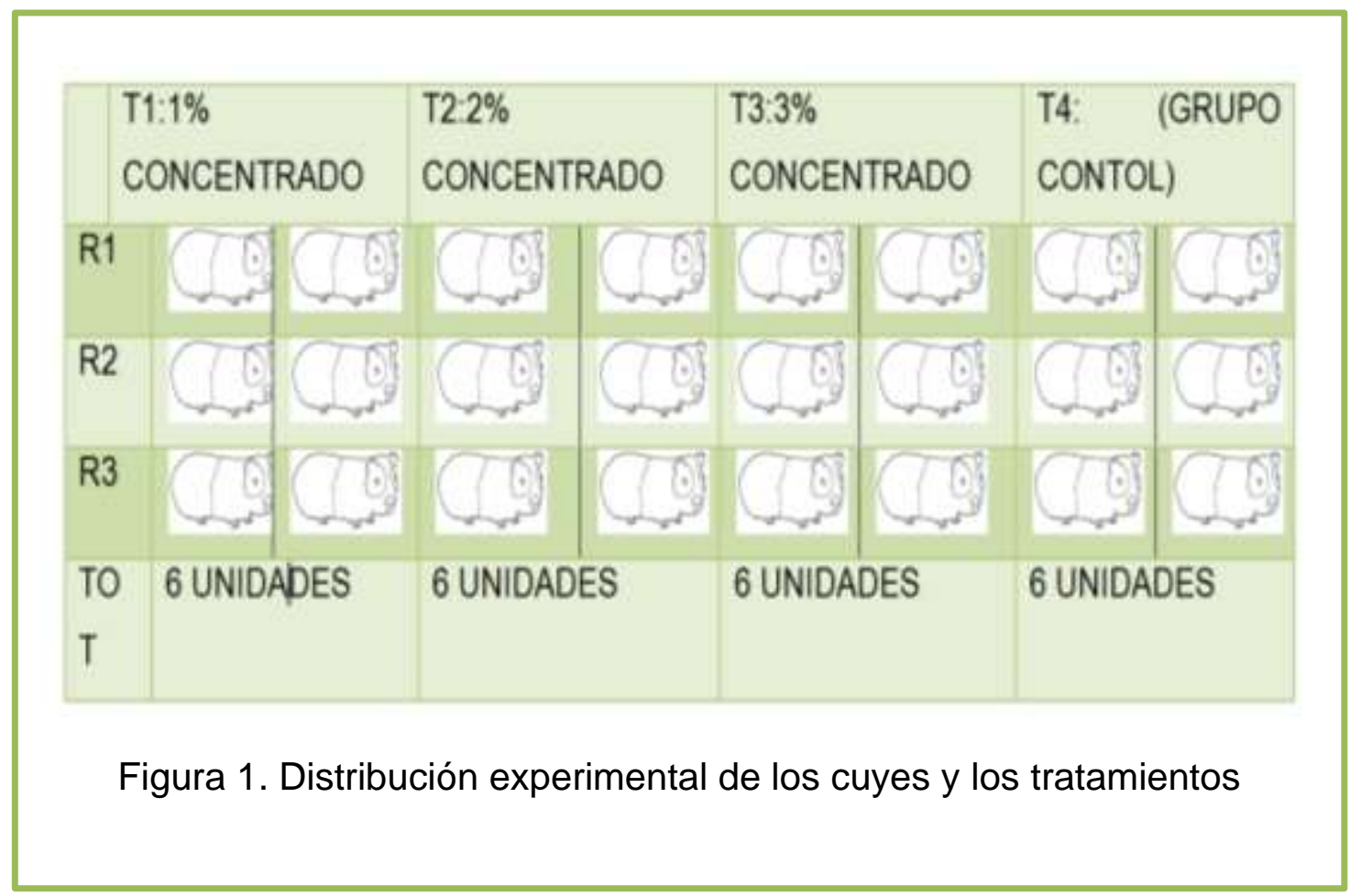

Tabla 1. Consumo y excreción promedios diarios (g) de los tratamientos por cuy, en materia seca

\begin{tabular}{ccccc}
\hline & $\begin{array}{c}\text { MF+CC + 1\% } \\
\text { de su PV }\end{array}$ & $\begin{array}{c}\text { MF+CC + 2\% } \\
\text { de su PV }\end{array}$ & $\begin{array}{c}\text { MF+CC + 3\% } \\
\text { de su PV }\end{array}$ & $\begin{array}{c}\text { MF ad } \\
\text { libitum }\end{array}$ \\
\hline Concentrado & 10.3 & 20.6 & 30.8 & 0 \\
Morera & 44.1 & 37.61 & 37.48 & 5026 \\
Total consumo & 54.4 & 59.21 & 68.28 & 50.26 \\
Excreción & 22.19 & 22.23 & 21.39 & 23.02 \\
\hline
\end{tabular}

CC: Concentrado comercial, MF: morera fresca, PV: peso vivo, Promedio de tres muestras analizadas en el laboratorio.

Fuente: Los autores.

Se aplicó un análisis de normalidad por medio de la prueba de Shapiro-Wilk y posteriormente un diseño completamente al azar y una prueba de comparación de medias (Tukey) con cuatro tratamientos y tres repeticiones aplicando la siguiente formula.

$$
y i j=\mu+T i+E i j
$$

$y i j$ : Variable aleatoria que representa la observación $j$-ésima del $i$-simo tratamiento 
$\mu$ : Efecto constante, común a todos los niveles. Media global.

$T i$ : Efecto del tratamiento $i$-ésimo. Es la parte de $y i j$ debida a la acción del nivel $i$ ésimo tratamiento, que será común a todos los elementos sometidos a ese nivel del factor (T1, T2, T3 y T4).

Eij: Variables aleatorias que engloban un conjunto de factores, es el error aleatorio.

Tabla 2. Análisis nutricionales de los tratamientos

\begin{tabular}{ccccc}
\hline Nutriente (\%) & $\begin{array}{c}\text { MF+CC + 1\% } \\
\text { de su PV }\end{array}$ & $\begin{array}{c}\text { MF+CC + 2\% de } \\
\text { su PV }\end{array}$ & $\begin{array}{c}\text { MF+CC + 3\% } \\
\text { de su PV }\end{array}$ & MF ad libitum \\
\hline Ceniza & $6.43 \pm 1.7$ & $6.52 \pm 1.8$ & $2.01 \pm 0.8$ & $13.71 \pm 2.2$ \\
Grasa & $2.00 \pm 1.5$ & $1.75 \pm 1.2$ & $0.54 \pm 0.1$ & $3.51 \pm 1.1$ \\
Proteína & $6.74 \pm 1.9$ & $5.45 \pm 1.7$ & $1.68 \pm 0.7$ & $10.67 \pm 2.4$ \\
Fibra cruda & $8.95 \pm 1.6$ & $8.80 \pm 25$ & $2.71 \pm 0.9$ & $18.35 \pm 3.1$ \\
ENN & $27.95 \pm 4.9$ & $24.20 \pm 4.9$ & $7.45 \pm 1.1$ & $48.56 \pm 4.7$ \\
FDN & $19.38 \pm 4.8$ & $18.40 \pm 3.3$ & $5.67 \pm 1.2$ & $38.00 \pm 4.2$ \\
FDA & $13.72 \pm 3.6$ & $13.44 \pm 2.8$ & $4.14 \pm 0.7$ & $28.00 \pm 3.7$ \\
\hline
\end{tabular}

ENN: Extracto no Nitrogenado, FDN: Fibra Detergente Neutra, FDA. Fibra Detergente Acida. CC: Concentrado comercial, MF: morera fresca, PV: peso vivo, Fuente. Promedio de tres muestras analizadas en el laboratorio.

\section{RESULTADOS Y DISCUSIÓN}

Se realizó el procesamiento de las muestras en el laboratorio de nutrición animal de la Universidad de los Llanos, los cuales fueron tabulados y sometidos a un análisis estadístico. En primera medida se realizaron pruebas de distribución de los datos (normalidad) en donde se evidencio que los datos siguen una distribución normal ya que su significancia en la prueba de Shapiro-Wilk fue mayor a 0.05 siendo este un parámetro que expresa la normalidad de las variables y encamina el estudio a realizar estadística paramétrica o no paramétrica (Otero et al., 2005).

\section{Coeficiente de digestibilidad (Cod) de la materia seca}

La determinación del Cod de MS es importante en nutrición animal debido a que calcula la cantidad de alimento consumido y excretado para establecer el aprovechamiento en términos de porcentajes, lo cual permite valorar la calidad de 
un alimento y sus componentes químicos. T1, T2, presentaron un mayor Cod de MS $(P<0.05)$ con 53.59 y $62 \%$ respectivamente mientras que el de $\mathrm{T} 4$ fue de $52.9 \%$, además se evidenció un incremento de Cod de MS de la morera a medida que se aumentaba el suministro de concentrado (Gráfico 1).

\section{Cod de la proteína}

La digestibilidad de la proteína en nutrición animal interviene como una sustancia primordial ya que forman parte de la estructura básica de tejidos (músculos, tendones, piel, uñas, etc.), durante todos los procesos de crecimiento y desarrollo, crean, reparan y mantienen los tejidos corporales; además desempeñan funciones metabólicas (actúan como enzimas, hormonas, anticuerpos.

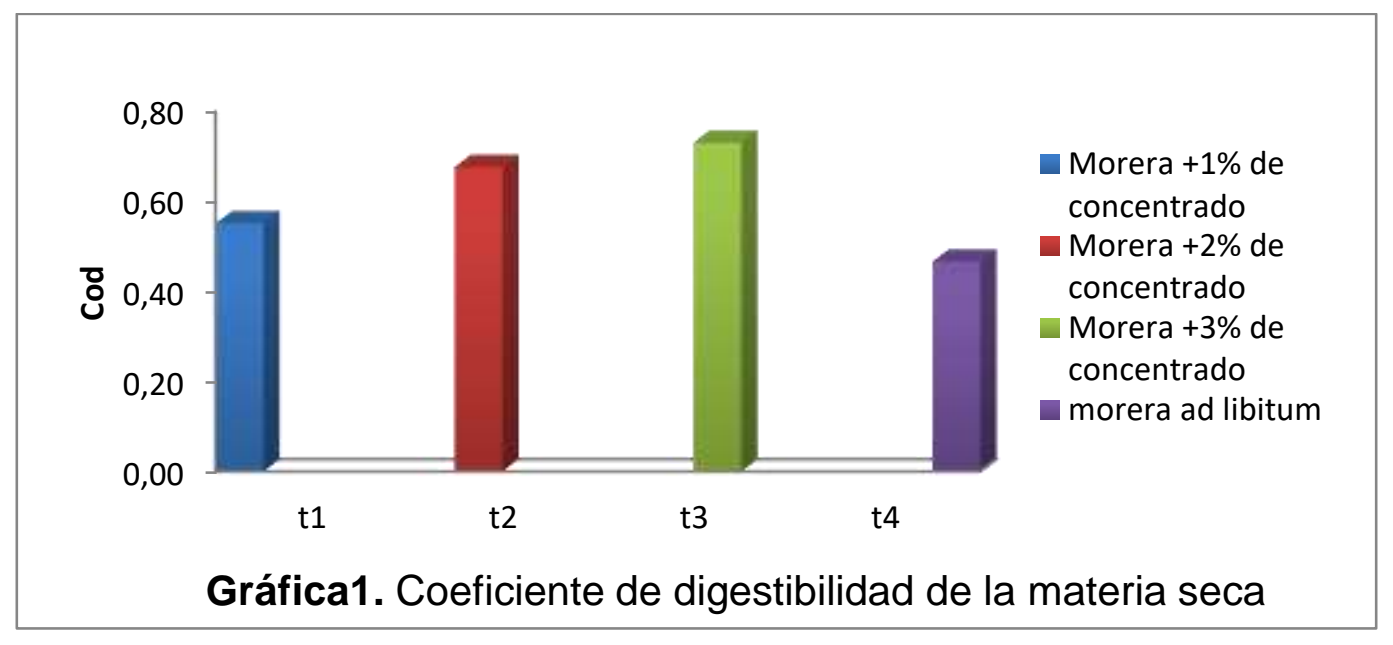

Con respecto al coeficiente de digestibilidad de proteína T2 y T3 proyectaron mayores valores de digestibilidad con 67 y $73 \%$ respectivamente $(P<0.05)$, entre los T1 y T4 se observó un comportamiento similar (Gráfica 2), lo que indica que a partir de un $2 \%$ de concentrado se evidencia aumento en la digestibilidad $(P>0.05)$ pero a un $3 \%$ de concentrado del peso vivo en materia seca (PVMS) no genera cambios importantes en la digestibilidad con respecto al $2 \%$ según la prueba de Tukey para la comparación de medias $(P>0.05)$.

Se observó que reemplazando el concentrado hasta un 2\% PVMS se puede aumentar la digestibilidad de la proteína desde un $46 \%$ (tratamiento control T4) a $73 \%$ (T3) no obstante el aumento del concentrado superior al 2\% PVMS no va a 
generar cambios significativos en la digestibilidad lo que acarrea perdida de la incorporación del mismo.

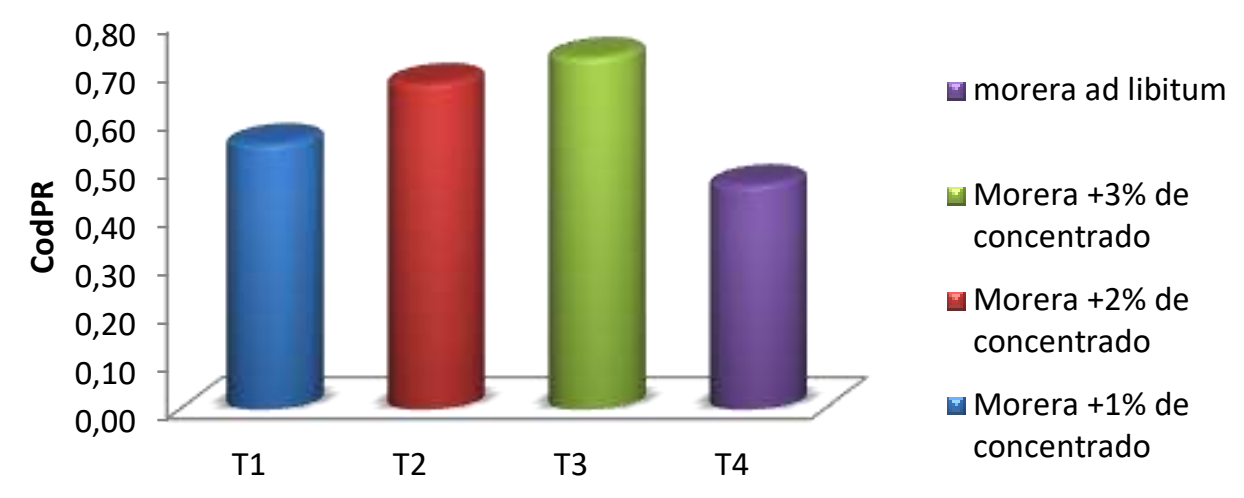

Gráfica 2. Coeficiente de digestibilidad de la proteína

\section{Cod de la grasa}

Los ácidos grasos en el alimento son necesarios en cantidad y en calidad, el aporte de las materias primas requiere presentar ácidos grasos esenciales y de buena biodisponibilidad, de la misma manera se debe garantizar una buena composición de grasas en canal, lo cual se obtiene con base en una buena alimentación. El aumento en la composición de ácidos grasos esenciales en canal está dado por el aporte de la morera y su composición nutricional con respecto a ácidos grasos en comparación con los concentrados comerciales (Garcia, 2005). En la asociación de morera en los cuyes no se presentaron diferencias significativas para ningún tratamiento $(P<0.05)$ (Gráfica 3) lo que indica que así se incorpore concentrado en los tratamientos no influirá en la digestibilidad de la materia seca, ahora bien, se verá un efecto positivo en la composición de ácidos grasos esenciales tal y como lo reporto (Garcia, 2005), y no es ajeno que el aporte de morera generará una canal con mejores características debido al cambio de ácidos grasos en composición.

\section{Cod de fibra cruda}

La fibra cruda es un componente esencial en la nutrición de los cuyes, FAO, (2005) reporta la necesidad de fibra en los cuyes es de 10 a $17 \%$ en la dieta. El rol de la 
fibra cruda en la digestión del cuy está enfocado a la absorción de los alimentos retardando los movimientos del contenido intestinal permitiendo una mayor eficiencia en la absorción de nutrientes; siendo en el ciego e intestino grueso donde se realiza la absorción de los ácidos grasos de cadenas cortas (Chauca, 1997). Los análisis bromatológicos de las materias primas suministradas en los alimentos datan de un $8.3 \%$ de aporte de la morera y un $18.35 \%$ en términos de fibra cruda lo que indica que la asociación de estos dos alimentos genera una simbiosis positiva nivelando el exceso de fibra de la morera y la carencia del concentrado.

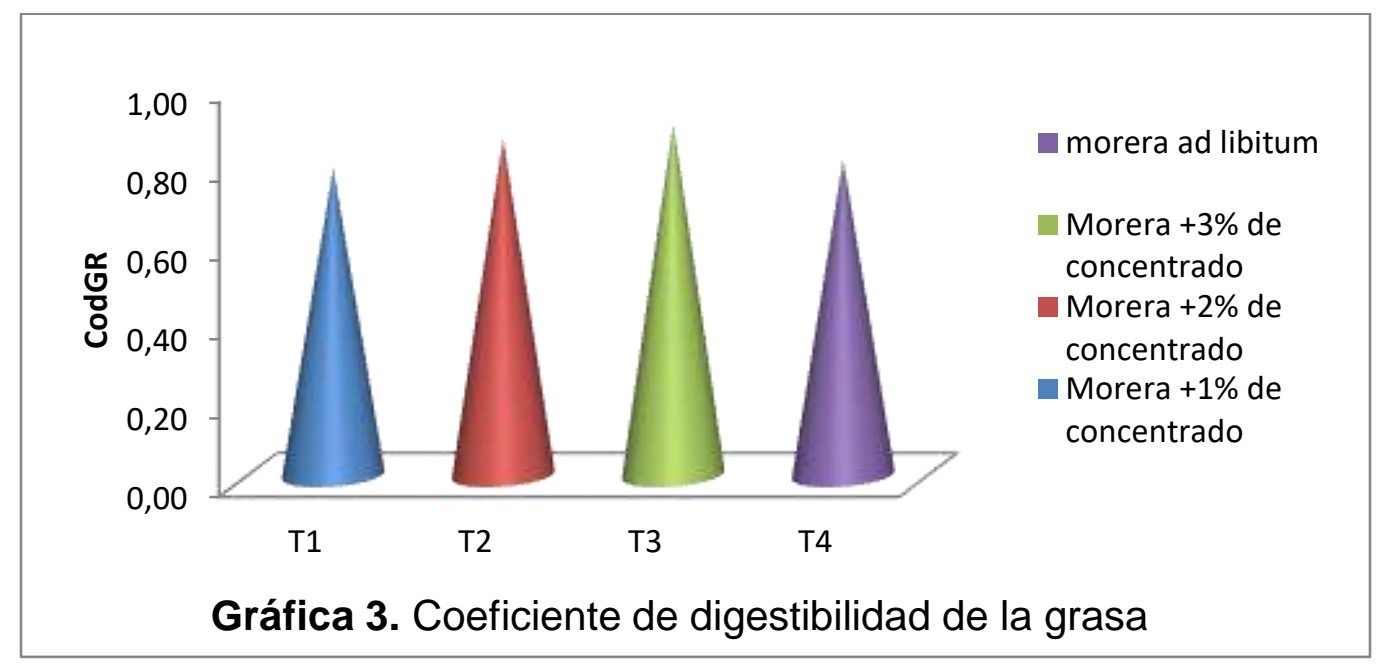

Los coeficientes de digestibilidad de fibra cruda (CodFC) no manifestaron diferencias significativas entre los tratamientos instaurados $(P<0.05)$ según el análisis de varianza, el tratamiento que mayor digestibilidad alcanzó fueron los tratamientos 1 y 2 con un $76 \%$ de CodFC mientras que el tratamiento 3 tuvo $74 \%$ y el tratamiento 4 un $73 \%$ de CodFC (Gráfica 4). Los CodFC se comportaron muy cercanos lo cual no genero impacto en los tratamientos con respecto a esta variable teniendo en cuenta que el aporte de la morera al concentrado comercial fue importante aumentando su carencia de fibra cruda con respecto a los requerimientos reportados.

\section{Cod de extracto no nitrogenado}

El extracto no nitrogenado es el componente energético del alimento, conformado por mono y disacáridos, los cuales son la parte soluble de la celulosa, lignina, 
almidón y todas las clases de azucares y materias solubles libres de nitrógeno (Bernal, 1993). El comportamiento de la morera y el reemplazo con diferentes niveles de concentrado en términos de CodENN evidencio diferencias estadísticamente significativas entre los tratamientos $(P>0.05)$. No obstante la tendencia de igualdad entre los tratamientos 2 y 3 continua presentándose, el T2 obtuvo un CodENN de 56\% y el T3 un CodENN de 64\% (Gráfica 5) siendo similar este parámetro para los dos tratamientos, mientras que T1 y T4 su CodENN fue diferente $(P>0.05) 44.3$ y 52\%, respectivamente, Se observó un efecto positivo en el reemplazo de morera por concentrado, sin embargo, después de un $2 \%$ PVMS de concentrado en la dieta no se observó variación, indicando de esta manera que el punto de equilibrio del reemplazo se encuentra en el T2, puesto que los resultados estadísticos $(P>0.05)$ después de un $2 \%$ PVMS de inclusión de concentrado en la dieta a base de morera no aumentan los CodENN.

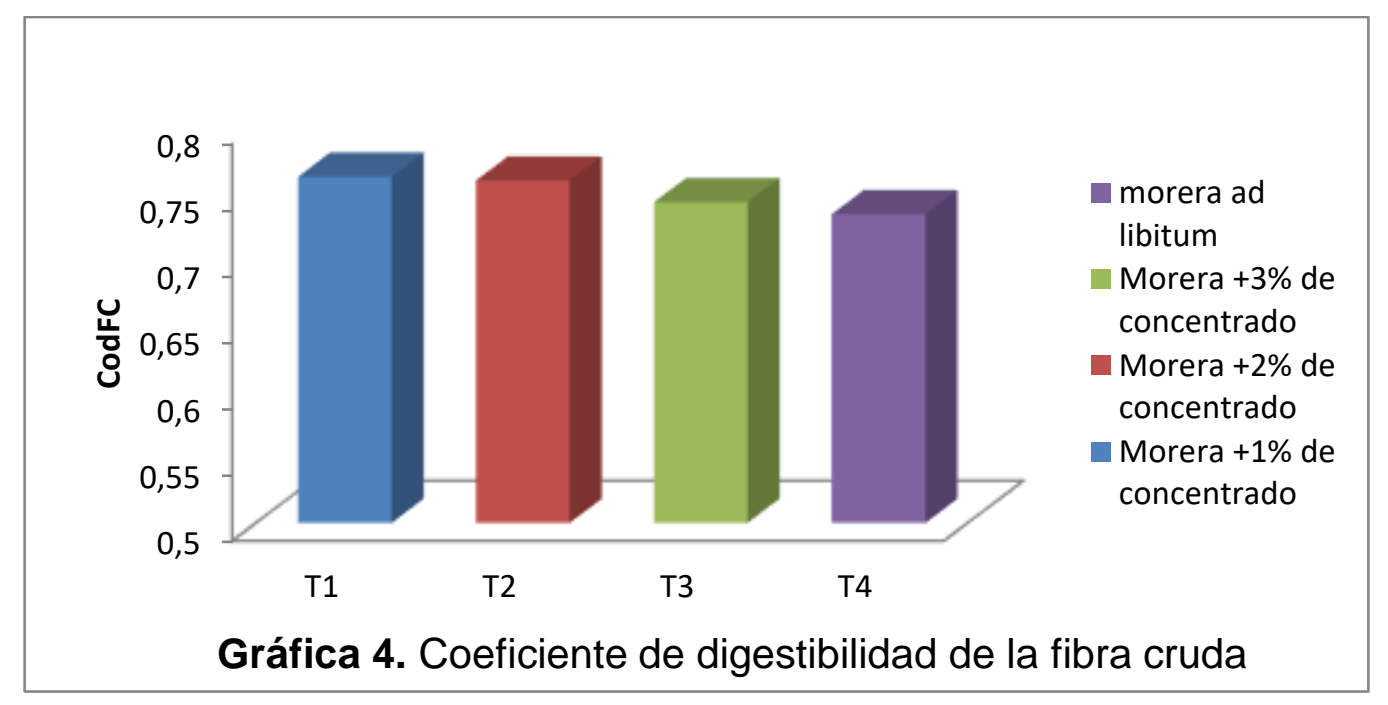

\section{Energía bruta (EB)}

Para la obtención de la energía bruta (EB) se realizó el cálculo con los porcentajes de digestibilidad de los nutrientes (proteína, fibra cruda, extracto no nitrogenado) expresados en gramos digeridos y multiplicados por 400 entendiéndose que un gramo del compuesto produce $400 \mathrm{Kcal}$ de energía bruta y la grasa es multiplicado por 900 ya que un gramo del nutriente digerido produce $900 \mathrm{Kcal}$ de energía bruta. (FAO, 2002). La EB en el presente trabajo se vio influenciada por los niveles de 
concentrado en la dieta. Entre los tratamientos, se observó una tendencia al aumento $(P>0.05)$ a medida que los niveles de concentrado se incrementaban en las dietas.

Como se observa en la Gráfica 6 y según los resultados del análisis de varianza, los tratamientos se vieron influenciados por la cantidad de concentrado instaurado en cada uno de los tratamientos y entre ellos se presentaron diferencias significativas $(P>0.05)$ el tratamiento que mayor cantidad de EB aporto fue el T3 con 389.46 Kcal de EB y el tratamiento control T4 fue el que menos cantidad de EB obtuvo con $350.06 \mathrm{Kcal}$. Lo anterior indica que el concentrado si influye positivamente en el aporte energético de la dieta hasta un 3\% de PVMS de concentrado por kg de peso vivo del animal.

\section{Energía digestible (ED)}

La energía digestible es aquella porción de la energía bruta a la cual se restan las pérdidas generadas en las heces y compone lo que fue digerido por el animal y será convertido en energía metabolizable (Bauza, 2012) Para hallar el contenido de ED en las dietas se partió de la premisa que un $\mathrm{kg}$ de NDT (nutrientes digestibles totales) equivale a 4.4 Mcal de ED (Cardona, 2001) por lo tanto se tomaron los datos de NDT del estudio y se realizó el cálculo pertinente.

La tendencia como era de esperarse es muy similar a la de energía bruta, observándose un impacto por la implementación de los tratamientos en el comportamiento de la ED $(P>0.05)$ no obstante, los tratamientos 2 y 3 no presentaron diferencias significativas entre ellos con 2.70 y 2.99 Mcal de ED respectivamente lo que indica que el aumento de más de un $2 \%$ PVMS de concentrado no genera diferencias estadísticas, aunque se observe una tendencia a aumentar.

\section{Energía metabolizable}

El contenido de energía metabolizable (EM) de un alimento corresponde a la cantidad de energía retenida por el organismo, representa la cantidad de energía 
presente en el alimento que el animal utiliza para sus diferentes necesidades. La EM se determina mediante la diferencia entre la EB del alimento que come el animal, y la energía presente en las heces y orina del animal (Correa, 2006). La EM no corresponde a un valor constante característico de la dieta o del ingrediente, sino que corresponde a una medida biológica propia del animal y depende de todos los factores que intervienen en la digestión y asimilación de nutrientes (Francesh, 2001). Para hallar el contenido de ED en las dietas se partió de la premisa que un $\mathrm{kg}$ de NDT (nutrientes digestibles totales) equivale a 3,8 Mcal de ED (Cardona, 2001)

Según el análisis de varianza para los tratamientos instaurados se presentaron diferencias significativas entre los mismos $(P>0.05)$ el tratamiento que mayor cantidad de energía metabolizable obtuvo fue el T3 con 2.58 Mcal de EM y el siguiente fue el T2 con 2.32 Mcal, entre estos dos tratamientos no se presentaron diferencias significativas por lo que se puede concluir que la incorporación de concentrado de más de 2\% PVMS de concentrado no generara cambios drásticos en la energía metabolizable.

En síntesis, se evidencio que la EB, ED y EM se comportaron de una manera similar y en las 3 variables el tratamiento 3 (T3) fue el que mayor valor presento seguido por el tratamiento 2 (T2) los cuales no presentaron diferencias estadísticamente significativas $(P>0,05)$, aunque se observe una leve diferencia numérica entre los dos tratamientos.

\section{Nutrientes digestibles totales (NDT)}

Los nutrientes digestibles totales son una de las variables más importantes debido a que reúne y resume todos los coeficientes de digestibilidad tratados anteriormente, en los tratamientos planteados su comportamiento fue diferente $(P>0,05)$ en donde a los tratamientos que mayores nutrientes digestibles totales se observaron en los tratamientos 2 y 3 siendo similar su comportamiento en esta variable. En la gráfica 6 que tras la incorporación de concentrado en las dietas fue aumentado paulatinamente la digestibilidad hasta un $2 \%$ PVMS de concentrado 
(T2), y en un reemplazo de 3\% PVMS de concentrado(T3) aumenta, pero no es significativo.

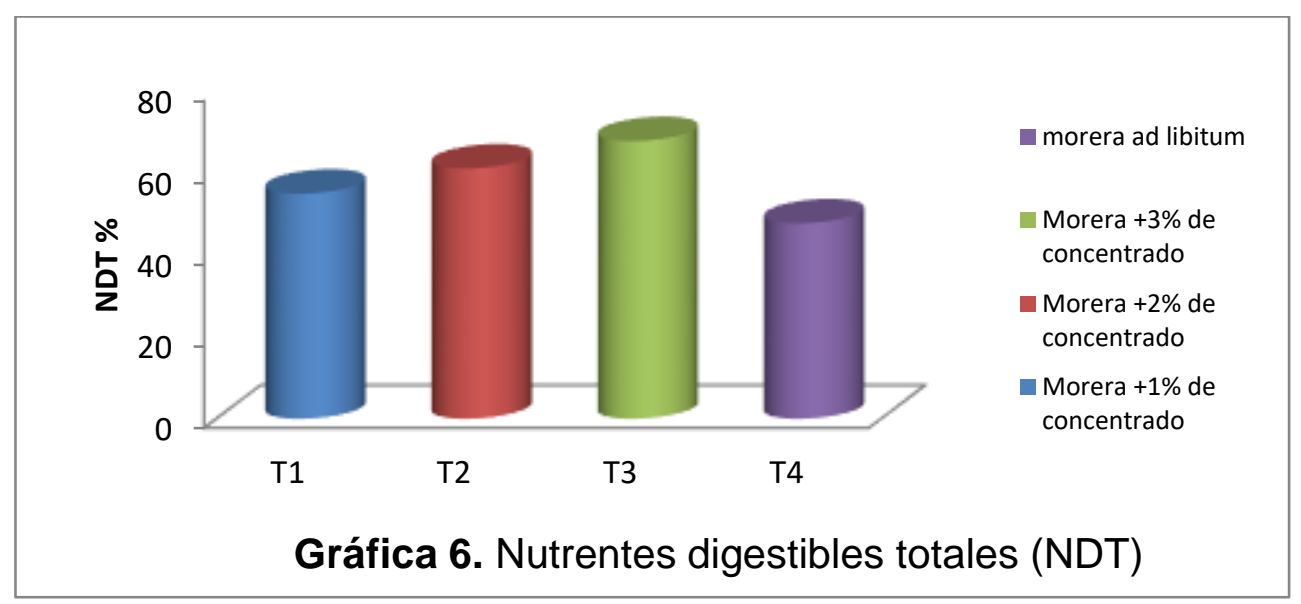

Los máximos NDT obtenidos se encuentran en $69 \%$ aproximadamente, comparado con lo encontrado por (Gary et al., 2012) se encontraron coeficientes de digestibilidad de $81 \%$ no obstante estos resultados son en base de harina de morera en Ecuador en donde se presentan condiciones totalmente distintas. La baja digestibilidad de la morera en el presente estudio puede estar dada por las condiciones de la planta y el suelo, la biodisponibilidad de nutrientes para el sector bajo las condiciones dadas. Para la zona son muy limitados los reportes con respecto a digestibilidad en cuyes.

Los valores obtenidos sirven de referencia para productores del sector que quieran potencializar su producción y reducir costos ya que un hallazgo importante fue que después de un 2\% PVMS de incorporación de concentrado no se presentan diferencias estadísticas pudiéndose utilizar la morera como especie adaptada al sector y el concentrado a un nivel óptimo que garantice una dieta balanceada.

\section{CONCLUSIONES}

Los estudios en cuyes son limitados en Colombia, sumado a esto no se ha elaborado un concentrado especial para esta especie por lo que se hace necesario alimentarlos con concentrado para conejos. El cuy ha tomado gran importancia en 
los últimos años en Colombia ya que es un animal resistente, precoz, de fácil manejo y prolifero, importante alternativa de seguridad alimentaria.

El estudio fue importante en el sentido de que se estimaron valores de referencia para el sector en términos de digestibilidad de la morera y el concentrado comercial en cuyes y se determinó la dieta que mejor comportamiento obtuvo la cual fue concentrado comercial al $2 \%$ del peso vivo en materia seca y morera a voluntad (T2) y $3 \%$ del peso vivo en concentrado y morera a voluntad (T3), a pesar que la que mayores valores de digestibilidad obtenidos fue el T3 el T2 no presenta diferencias significativas según el estudio pudiéndose utilizar sin disminuir drásticamente la digestibilidad y ahorrando un $1 \%$ de concentrado por animal representado un ahorro importante.

Evidentemente es necesario realizar y reportar estudios que involucren esta especie con diferentes materias primas, con el tiempo el cuy será una alternativa de alimentación económica, nutritiva y rentable.

\section{REFERENCIAS BIBLIOGRÁFICAS}

1. Barreyro A. Evaluación de la digestibilidad in vivo de raciones para becerros en crecimiento conteniendo desechos de la industrialización de los cereales. Universidad Autónoma de Querétaro. 2000.

Bauza, R. Curso en nutrición animal. 2012.

Benavides. Manejo y utilización de morera (Morus alba) como forraje. Agroforestería las Américas, 2 (7): 4.1995

Bernal. Análisis de alimentos. Bogotá. 1993.

Cardona, H. J. EL modelo NRC 2001. Sección de nutrición animal. 2001.

Carvajal, G. Valor nutricional de la carne de res. Corporación de fomento ganadero. 2001.

Chauca. Producción de cuyes (Cavia porcellus). Perú. 1997.

Correa, K. Determinación de energía metabolizable en aves. Universidad de Chile. 2006.

9. Cruz, E., Villarreal, H., Tapia, M., Nieto, M. Metodologías de digestibilidad in vivo e in vitro para ingredientes y dietas para camarón. México. 2008.

10. FAO. Nutrición humana en el mundo en desarrollo. En: M. C. Latham, Nutrición humana en el mundo en desarrollo. Roma. 2002.

11. FAO. Producción de cuyes. 2005.

12. Fedegan. Estudio sectorial carne bovina en Colombia (2009-2011). Superintendencia de Industria y Comercio. 2011.

13. Francesh, M. Sistemas para la valoración energética de los. Arch. Latinoam. Prod. Anim. 2001.

14. Garcia, I. D. Evaluación del contenido de ácidos grasos en la canal de cuyes. Bogotá. 2005.

15. Gary, A.-B., Sánchez, R., Meza, A., Meza, j., Franco-Suescum, N., Liuba-Delfini, G. Digestibilidad in vivo de forrajeras arbustivas tropicales para la alimentación de cuyes (Cavia porcellus Linnaeus), en el litoral ecuatoriano. Veterinaria y Zootecnia, ISSN $2011-5415$. 2012.

16. Hurtado, D. I., Nocua, S., Narváez-Solarte, W., Vargas-Sánchez, J. E. Valor nutricional de la morera (Morus sp.), matarratón (Gliricidia sepium), pasto india (Panicum máximum) y arboloco (Montanoa quadrangularis) en la alimentación de cuyes (Cavia porcellus). vet. zootec., 6 (1): 56-65. 2012.

17. Medina, M., Moratinos, P., García, D., Cova, L. La morera (Morus spp.) como recurso forrajero Avances y consideraciones de investigación. Zootecnia Trop., 27 (4): 343-362. 2009.

18. Otero, J. V., Herrarte Sánchez, A., Medina Moral, E. Análisis de la Varianza. 2005.

19. Pajares, C. Reproducción y Manejo Reproductivo en Cuyes. Sistema de revisiones en investigación veterinaria de San Marcos, p 9. 2009.

20. Perez, S., Gomez, G., Barriga, H. Utilización de la remolacha como marcador digestivo en conejos para determinar el tiempo de la digestión de esta especie menor. Universidad Francisco de Paula Santander, 16 p. 2010.

21. Pond, Church. Fundamentos de nutrición y alimentación de animales. En Fundamentos de nutrición y alimentación de animales. México: Editorial Limusa, S.A de. C.V. Grupo Noriega Editores. 438 p. 1994.

22. Sanchez, M., Carcelén F., Ara M., González R., Quevedo W., Jiménez R. Efecto de la suplementación de ácidos orgánicos sobre parámetros productivos del cuy (Cavia porcellus). Rev. investig. vet. Perú, 25 (3): 381-389. 2014.

23. Santos, V. G. Importancia del cuy y su competitividad. En: XX Reunión ALPA, XXX Reunión APPA-Cusco-Perú. 2007. 